\title{
Computing Isophotes on Free-form Surfaces based on Support Function Approximation
}

\author{
M. Aigner ${ }^{1}$, L. Gonzalez-Vega ${ }^{2}$, B. Jüttler ${ }^{1}$, and M. L. Sampoli ${ }^{3}$ \\ 1 Johannes Kepler University, Linz, Austria \\ 2 University of Cantabria, Santander, Spain \\ 3 University of Siena, Italy
}

\begin{abstract}
The support function of a free-form-surface is closely related to the implicit equation of the dual surface, and the process of computing both the dual surface and the support function can be seen as dual implicitization. The support function can be used to parameterize a surface by its inverse Gauss map. This map makes it relatively simple to study isophotes (which are simply images of spherical circles) and offset surfaces (which are obtained by adding the offsetting distance to the support function).

We present several classes of surfaces which admit a particularly simple computation of the dual surfaces and of the support function. These include quadratic polynomial surfaces, ruled surfaces with direction vectors of low degree and polynomial translational surfaces of bidegree $(3,2)$. In addition, we use a quasi-interpolation scheme for bivariate quadratic splines over criss-cross triangulations in order to formulate a method for approximating the support function. The inverse Gauss maps of the bivariate quadratic spline surfaces are computed and used for approximate isophote computation. The approximation order of the isophote approximation is shown to be 2 .
\end{abstract}

\section{Introduction}

This paper is devoted to the use of support functions and dual implicitization in order to deal with the problem of computing isophotes of free form surfaces. Like reflection lines and highlight lines, isophotes are very useful tools for shape interrogation, see $[11,12]$. They are defined as lines of equal light intensity and they are used to detect and visualize small surface irregularities and discontinuities that can not be seen by other means like, for example, a shaded surface image.

The first use of isophotes in this context is due to [22]. Isophotes appear also in other contexts such as Image Processing for the so called image interpolation (e.g. [18, 28]), Computer Vision for object detection (e.g., [17]) or in the study of feature sensitive mathematical morphology of surfaces where an isophotic metric has been introduced [25].

Dual implicitization (or support function computation) provides an alternative way to represent curves and surfaces that allows, in many cases, to analyze 
and to manipulate the considered curves and surfaces $[26,9]$. In this paper we show several concrete cases (quadratic polynomial surfaces, ruled surfaces with polynomial direction vectors of low degree, polynomial translational surfaces of low degree or some special cubics) where the dual implicit equation is very easy to compute explicitly in terms of rational functions and square roots. Using dual implicitization and the inverse Gauss map then isophotes are easy to characterize for these cases.

In order to compute the isophotes on free form surfaces in the general case we propose to compute a support function approximation through the computation of a piecewise quadratic approximation for the considered free form surface. This support function then defines the inverse Gauss maps which are used to determine the isophotes for the considered free form surface.

The remainder of this paper is organized as follows. After recalling the notions of dual surfaces and support functions of free-form surfaces, the second section discusses the support functions of several particular classes of surfaces: quadratic polynomial surfaces, rational ruled surfaces with direction vectors of low degree, polynomial translational surfaces of low degree, and special cubic polynomial surfaces. Section 3 uses the support function to analyze offset surfaces, isophotes and contour generators. Based on a simple representation for the inverse Gauss map, it presents results on exact rational paramaterizations of offsets and on parametric represetations of isophotes, in both cases for special classes of surfaces. Section 4 discusses the case of general free-form surfaces. We propose to approximate the support function via quasi-interpolation by piecewise quadratic surfaces and use the result for approximate isophote computation. Finally we analyze the convergence of the method.

\section{Dual implicitization}

We recall the notions of the dual surface and the support function of a rational surface. In the second part we discuss several classes of surfaces whose support function can be expressed by using solely square roots and rational functions.

\subsection{Dual surface and support functions}

We consider a non-developable polynomial or rational surface $\mathbf{p}: \Omega \rightarrow \mathbb{R}^{3}$ with domain $\Omega \subseteq \mathbb{R}^{2}$. Its partial derivative vectors with respect to the surface parameters $u, v$ will be denoted with $\mathbf{p}_{u}$ and $\mathbf{p}_{v}$, respectively.

Consider the three equations

$$
\begin{aligned}
h-\mathbf{p}(u, v)^{\top} \mathbf{n} & =0, \\
\mathbf{p}_{u}(u, v)^{\top} \mathbf{n} & =0, \\
\mathbf{p}_{v}(u, v)^{\top} \mathbf{n} & =0 .
\end{aligned}
$$

If these equations are satisfied by non-trivial values of $h$ and $\mathbf{n}$, then the plane with normal $\mathbf{n}$ and distance $h /\|\mathbf{n}\|$ to the origin is the tangent plane of the surface at its point $\mathbf{p}(u, v)$. 
This system is homogeneous with respect to $h$ and $\mathbf{n}$. Eliminating $u$ and $v$ from (1)-(3) leads to a single equation of the form

$$
F(\mathbf{n}, h)=\sum_{i=0}^{d} h^{i} f_{m-i}(\mathbf{n})=0
$$

for certain values of the total degree $m$ and the degree $d$ with respect to $h$. This equation is homogeneous of degree $m$ with respect to $h$ and $\mathbf{n}$, since the original equations (1)-(3) were homogeneous. Consequently, the functions $f_{m-i}$ are homogeneous polynomials of degree $m-i$ in $\mathbf{n}$.

Eq. (4) is the dual implicit equation of the surface $\mathbf{p}$, as it is satisfied by the coefficients of the tangent planes of the surface. We refer to the process of computing this equation as dual implicitization.

In order to visualize it, one may consider the surface

$$
F\left((x, y, z)^{\top}, 1\right)=0
$$

which is obtained by substituting $h=1$ and $\mathbf{n}=(x, y, z)^{\top}$. This is the dual surface (see [13]), which is generated by applying the polarity with respect to the unit sphere to the tangent planes of $\mathbf{p}$.

Hoschek [13] uses the polarity with respect to the imaginary unit sphere. We shall use the standard unit sphere instead, see e.g. [10]. In this case, a point $\mathbf{q}$ is mapped to the plane $\mathbf{q} \cdot \mathbf{x}=1$ and vice versa, and the center of the sphere is mapped to the plane at infinity.

We are particularly interested in surfaces where it is possible to solve the two equations (2)-(3) for $u, v$ by using rational operations and square roots. After substituting the result into (1), the variable $h$ can be expressed by a function

$$
h=H(\mathbf{n})
$$

of $\mathbf{n}$. The function $H$ may possess different branches, if square roots are involved. For a given normal $\mathbf{n}$, the different roots of $F(\mathbf{n}, h)=0$ are the different possible values of $H$.

The function $H$ is a 1-homogeneous function, i.e.

$$
H(\lambda \mathbf{n})=\lambda H(\mathbf{n}),
$$

since the elimination procedure preserves the homogeneity of the original system. The equation

$$
H(\mathbf{n})=1
$$

is another implicit representation of the dual surface (5).

The restriction of $H$ to the unit sphere is the support function of the surface. This function is always odd,

$$
H(-\mathbf{n})=-H(\mathbf{n}),
$$

since $H$ is homogeneous. The value(s) of $H(\mathbf{n})$ is (are) the support distance(s) of the tangent plane(s) with unit normal $\mathbf{n}$ (cf. see $[9,26,10]$ ).

We describe several classes of surfaces leading to certain special values of the degrees $d$ and $m$ which admit closed form solutions of (4) with respect to $h$. 


\subsection{Quadratic polynomial surfaces}

We recall results from [10, Example 2] on the support function of non-developable quadratic polynomial surfaces (triangular Bézier surfaces of degree 2), see also [16]. These surfaces possess an affine classification, which is described in [4,21]. We are mainly interested in non-developable surfaces. Developable quadratic polynomial surfaces are planes, parabolic cylinders or quadratic cones.

If the components of $\mathbf{p}$ are quadratic polynomials in $u$ and $v$, then - in the generic case - the dual implicit equation has the form (4) with $d=1$ and $m=3$. Consequently, the support function $H$ is an odd rational function

$$
H=-f_{3}(\mathbf{n}) / f_{2}(\mathbf{n})
$$

which is the quotient of two homogeneous polynomials $f_{3}$ and $f_{2}$ of degree 3 and degree 2, respectively. The dual surface is a cubic monoid surface (see [15]) with a threefold point at the origin.

\subsection{Ruled surfaces with polynomial direction vectors of low degree}

Next we consider ruled surfaces,

$$
\mathbf{p}(u, v)=\mathbf{q}(u)+v \mathbf{w}(u)
$$

where the directrix $\mathbf{q}(u)$ is a rational curve and the direction vector of the generators are described by a polynomial function

$$
\mathbf{w}(u)=\sum_{i=0}^{k} u^{i} \mathbf{w}_{i}
$$

with certain real coefficient vectors $\mathbf{w}_{i} \in \mathbb{R}^{3}$. The two equations (2), (3) take the form

$$
\left[\mathbf{q}^{\prime}(u)+v \mathbf{w}^{\prime}(u)\right]^{\top} \mathbf{n}=0 \quad \text { and } \quad \mathbf{w}(u)^{\top} \mathbf{n}=0 .
$$

We analyze the cases $k=1$ and $k=2$.

$-\underline{k=1}$ : The two equations (13) are linear with respect to $v$ and $u$, respectively, and the second equation does not involve $v$. One may easily express both $u$ and $v$ by rational functions of $\mathbf{n}$.

The dual implicit equation takes the form (4) with $d=1$. The support function of the surface is an odd rational function.

$-\underline{k=2:}$ The first equation is linear in $v$ and it can be used to express it as a rational function of $\mathbf{n}$ and $u$. The second equation is a quadratic equation for $u$, which can be solved by using one square root. Note that its argument is a homogeneous quadratic form of $\mathbf{n}$.

The dual implicit equation takes the form (4) with $d=2$. The support function of the surface can be expressed as

$$
H(\mathbf{n})=R\left(\mathbf{n}, \pm \sqrt{\mathbf{n}^{\top} D \mathbf{n}}\right)
$$

where $R$ is a homogeneous rational function and $D$ is a symmetric $3 \times 3$ matrix. 
Consequently, rational ruled surfaces with linear and quadratic direction vectors are special instances of the surfaces studied in [10] and [1], respectively.

\subsection{Polynomial translational surfaces of low degree}

A translational surface is generated by translating a moving curve along a fixed curve. The general parametric representation of a rational translational surface is

$$
\mathbf{p}(u, v)=\mathbf{q}(u)+\mathbf{r}(v)
$$

where $\mathbf{q}$ and $\mathbf{r}$ are rational curves. The last two equations (2) and (3) take the form

$$
\mathbf{q}^{\prime}(u)^{\top} \mathbf{n}=0 \quad \text { and } \quad \mathbf{r}^{\prime}(v)^{\top} \mathbf{n}=0 .
$$

We analyze the special case where both $\mathbf{q}$ and $\mathbf{r}$ are polynomial curves of low degree (at most cubic). We assume that $\mathbf{q}$ is a cubic curve and study the cases of quadratic and cubic curves $\mathbf{r}$ separately.

- $\mathbf{r}$ is quadratic: The first equation in (16) is quadratic with respect to $u$ and the coefficients are linear in the coordinates of $\mathbf{n}$. The second equation (16) is even just linear in $v$, hence $u$ and $v$ can immediately be computed from $\mathbf{n}$ using rational functions and one square root.

The dual implicit equation takes the form (4) with $d=2$ and $m=6$. The support function of the surface can be expressed as

$$
H(\mathbf{n})=R\left(\mathbf{n}, \pm \sqrt{\mathbf{n}^{\top} D \mathbf{n}}\right)
$$

where $R$ is a homogeneous rational function with a numerator of degree 4 and a denominator of degree 3 , and $D$ is a symmetric $3 \times 3$ matrix. Consequently, polynomial translational surfaces of degree $(3,2)$ are special instances of the surfaces studied in [1].

- $\underline{\mathbf{r}}$ is cubic: Both equations in (16) are now quadratic. The parameters $u$ and $v$ can immediately be computed from $\mathbf{n}$ using rational functions and two square roots.

The dual implicit equation takes the form (4) with $d=4$ and $m=12$. The support function of the surface can be expressed as

$$
H(\mathbf{n})=R\left(\mathbf{n}, \pm \sqrt{\mathbf{n}^{\top} D_{1} \mathbf{n}}, \pm \sqrt{\mathbf{n}^{\top} D_{2} \mathbf{n}}\right) .
$$

It is a homogeneous rational function with a numerator of degree 5 and a denominator of degree 4 , and $D_{1}$ and $D_{2}$ are two symmetric $3 \times 3$ matrices.

Example 1. The computation of the support function of the translational surface

$$
\mathbf{p}(u, v)=\left(u, v, u^{2}+v^{3}\right)^{\top}
$$

which is generated by translating the cubic parabola along the quadratic parabola gives

$$
H(\mathbf{n})=\frac{1}{36} \frac{-9 x^{2} \pm 8 \sqrt{3} \sqrt{-z y} y}{z}
$$

where $\mathbf{n}=(x, y, z)^{\top}$. 


\subsection{Special cubic polynomial surfaces}

Finally we mention two special classes of cubic surfaces whose support function can be computed explicitly simply by using square roots. A general polynomial cubic surface has a parametric representation of the form

$$
\mathbf{p}(u, v)=\sum_{i=0}^{3} \sum_{j=0}^{3-i} \mathbf{a}_{i, j} u^{i} v^{j}
$$

with real coefficients $\mathbf{a}_{i, j} \in \mathbb{R}^{3}$. If the coefficients satisfy either

$$
\mathbf{a}_{2,1}=\mathbf{a}_{2,0}=\mathbf{a}_{1,1}=\mathbf{a}_{0,2}=\mathbf{a}_{0,1}=0
$$

or

$$
\mathbf{a}_{2,1}=\mathbf{a}_{1,2}=\mathbf{a}_{0,3}=0,
$$

then the two equations (2), (3) can be used to express $u$ and $v$ as functions of $\mathbf{n}$ using rational functions and square roots. Consequently, the support functions of these surfaces involve only rational expressions and square roots, but the arguments of the roots are now polynomials with a higher degree than two.

\section{Offsets, isophotes and contour generators}

We discuss the inverse Gauss map of surfaces with odd support functions and address the existence and computation of rational PN (Pythagorean normal) parameterizations. Finally we analyze the parameterization of isophotes with the help of the inverse Gauss map.

\subsection{The inverse Gauss map}

Any 1-homogeneous function $H$ satisfies

$$
\nabla H(\lambda \mathbf{n})=\nabla H(\mathbf{n})
$$

i.e., the gradient field is constant along the lines through the origin. Indeed, applying the gradient operator to (7) gives (24), where both sides of the equation are multiplied by $\lambda$.

For any point $\mathbf{n}$, the scaled point $\mathbf{n}_{0}=\mathbf{n} / H(\mathbf{n})$ lies on the dual surface, since it satisfies

$$
H\left(\mathbf{n}_{0}\right)=H\left(\frac{\mathbf{n}}{H(\mathbf{n})}\right)=\frac{1}{H(\mathbf{n})} H(\mathbf{n})=1,
$$

see (8). Moreover the normal vector of the dual surface at $\mathbf{n}_{0}$ is $(\nabla H)(\mathbf{n})$, due to (24). The equation of the tangent plane of the dual surface at $\mathbf{n}_{0}$ is

$$
(\nabla H)(\mathbf{n}) \cdot \mathbf{x}=1
$$


since

$$
(\nabla H)(\mathbf{n}) \cdot \frac{\mathbf{n}}{H(\mathbf{n})}=\left.\frac{1}{H(\mathbf{n})} \frac{\mathrm{d}}{\mathrm{d} t} H((1+t) \mathbf{n})\right|_{t=0}=\left.\frac{1}{H(\mathbf{n})} \frac{\mathrm{d}}{\mathrm{d} t}(1+t)\right|_{t=0} H(\mathbf{n})=1 .
$$

Now we apply the polarity with respect to the unit sphere to the tangent plane (26). This leads to the corresponding point $\nabla H(\mathbf{n})$ of the original surface, such that the vector $\mathbf{n}$ is a normal vector of the surface. Consequently, the mapping

$$
\Gamma: \quad \mathbb{S}^{2} \rightarrow \mathbb{R}^{3}: \quad \mathbf{n} \rightarrow(\nabla H)(\mathbf{n})
$$

is the inverse Gauss map of the surface with the 1-homogeneous support function $H$, see $[9,10]$.

Example 2. The inverse Gauss maps of the translational surface from Example 1 are

$$
\Gamma(\mathbf{n})=\left(-\frac{x}{2 z},-\frac{\sqrt{3} y}{ \pm 3 \sqrt{-z y}}, \frac{ \pm 9 \sqrt{-z y} x^{2}+4 \sqrt{3} z y^{2}}{ \pm 36 z^{2} \sqrt{-z y}}\right)^{\top},
$$

where $\mathbf{n}=(x, y, z)$. This expression is constant along the lines through the origin, hence it can also be evaluated at non-normalized normal vectors.

\section{2 $\quad$ PN parameterizations and offset surfaces}

Consider a rational parameterization $\nu: \Omega \rightarrow \mathbb{S}^{2}$ of the sphere with parameters $(u, v) \subset \Omega \subseteq \mathbb{R}^{2}$, cf. [5]. The parameterization of the surface

$$
\mathbf{q}=\Gamma \circ \nu
$$

which is obtained by composing $\nu$ with the inverse Gauss map, is a PN (Pythagorean Normal vector) parameterization of the given surface, see [23]. It has an associated polynomial field of (non-normalized) normal vectors, such that their length is equal to the square of another polynomial, hence the components of the polynomial normals satisfy a Pythagorean condition.

A general construction and a design methodology for rational PN parameterizations have been described in [23], based on the dual representation and control structure of rational surfaces. These surfaces have also been studied in the frame of Laguerre geometry [20]. As an advantage of rational PN surfaces, the offset surfaces are again rational. They can be obtained either by adding the offsetting distance to the support function or by adding constant multiples of the rational unit normals to the PN parameterization.

Clearly, surfaces with rational support functions have rational PN parameterizations, since their inverse Gauss maps $\Gamma$ are again rational. This is true both for odd rational support functions, where $\Gamma$ takes the form (27), and for general rational support functions, see [10]. As observed in Section 2.2, this case includes quadratic polynomial surfaces and rational ruled surfaces with polynomial direction vectors. 
In addition, surfaces with support functions of the form (14) or (17), where $R$ is a rational function and the matrix $D$ has rank 3 , can be equipped with rational PN parameterizations. This is proved by describing an algorithm for generating the parameterization in [1], where support functions of this type have been derived by studying quadric surfaces. It is shown that the parameterization of these surfaces is closely related to the analysis of the intersection of two quadrics in four-dimensional space, which forms a del Pezzo surface.

Based on the results in Section 2.2 we note following fact.

Proposition 1. Non-developable ruled surfaces with polynomial direction vectors of degree less than three and non-developable polynomial translational tensorproduct surfaces of degree $(3,2)$ possess rational PN parameterizations.

Proof. The PN parameterizations can be constructed as follows. First we compute the support function, which gives functions of the form (14). Second we apply the parameterization technique in [1]. This technique is not limited to regular matrices $D$. It is possible to use it for surfaces with singular quadratic forms, too. (See also the example below.)

PN parameterizations of rational ruled surfaces where also discussed in [24, 19]. The first paper [24] observes that these parameterizations always exist. It also presents an algorithm for parameterization for the case of direction vectors of low degree (less than 3). The second paper [19] shows how to find parameterizations over the field of complex numbers which are, however, not directly useful for geometric computing.

PN parameterizations of translational surfaces have not been discussed previously.

Example 3. In order to find a PN parameterization of the translational surface from Example 1 with the inverse Gauss maps (28), we consider the two quadrics

$$
x^{2}+y^{2}+z^{2}=1 \text { and } y z=w^{2}
$$

in four-dimensional $(x, y, z, w)$-space. We generate a rational parameterization $(x(u, v), \ldots, w(u, v))$ of their intersection 2 -surface. Then we may substitute it into (28) and replace the square roots by $|w(u, v)|$.

The substitution $x=Z, y=\frac{1}{2} \sqrt{2}(X-Y), z=\frac{1}{2} \sqrt{2}(X+Y), w=W$ transforms the two quadrics (30) into

$$
X^{2}+Y^{2}+Z^{2}=1 \text { and } X^{2}-Y^{2}=W^{2} .
$$

We apply a central projection with center $(1,0,0,1)$ into the hyperplane $X=0$ and obtain the cubic surface

$$
W Z^{2}-W+W Y^{2}+Y^{2}+W^{2}=0
$$

It contains the line $W=Y=0$ which passes through two singular points (at $Z= \pm 1$ ) of the surface. One may now parameterize this cubic surface using the 
family of conics which are obtained as intersections with planes through the line. After a short computation one arrives at

$$
\begin{gathered}
x=\left(-2+2 u^{2} v^{2}-2 u v^{2}-2 u\right) / N, \\
y=\frac{1}{2} \sqrt{2}\left(2+u v^{2}+u\right) u(v+1)^{2} / N, \\
z=\frac{1}{2} \sqrt{2}\left(2+u v^{2}+u\right) u(v-1)^{2} / N, \\
N=u^{2} v^{4}+4 u^{2} v^{2}+u^{2}+2 u v^{2}+2 u+2
\end{gathered}
$$

which defines a rational parameterization of the sphere of degree $(3,4)$.

\subsection{Isophotes}

Recall that an isophote (a line of constant brightness) on a $C^{1}$ smooth surface is the set of all points where

$$
\angle\left(\mathbf{p}_{u} \times \mathbf{p}_{v}, \mathbf{d}\right)=\angle(\mathbf{n}, \mathbf{d})=\phi_{0}
$$

where $\mathbf{d}$ is the direction of the incoming light and $\phi_{0}$ is the (constant) angle between the surface normal and the light direction, see [14]. For given values of $\mathbf{d}$ and $\phi_{0}$, the unit normals along the isophotes form a circle on the unit sphere.

In particular, if $\phi_{0}=\pi$, then the isophote is the contour generator of the surface with respect to the parallel projection with direction $r$. The corresponding normals form the great circle on the sphere which lies in the plane with normal $\mathbf{d}$ through the origin. The contour generator contains the shadow boundary with respect to illumination with direction $\mathbf{d}$.

Since circles possess a rational parameterizations, one obtains that isophotes on surfaces with rational support functions are rational curves. This is equivalent to [23, Theorem 3.3], which analyzes isophotes on surfaces possessing PN parameterizations whose unit normals define birational parameterizations of the sphere. This class of surfaces is identical to the set of surfaces with rational support functions.

In particular we analyze the case of quadratic polynomial surfaces, which have rational support functions of degree $(3 / 2)$. The inverse Gauss map is a rational function of degree (4/4). We obtain the following result.

Proposition 2. The isophotes (resp. contour generators) on non-developable quadratic polynomial surfaces are rational curves of degree 8 (resp. 4), which correspond to rational curves of degree 4 (resp. 2) in the parameter domain of the surface.

Proof. The isophotes are obtained by applying the inverse Gauss map to spherical circles, which possess rational quadratic parameterizations. The contour generators are found by applying the inverse Gauss map to lines, since these project into great circles on the sphere (by a central projection with respect to the origin) and the inverse Gauss map gives the same point for all points along a line through the origin. 

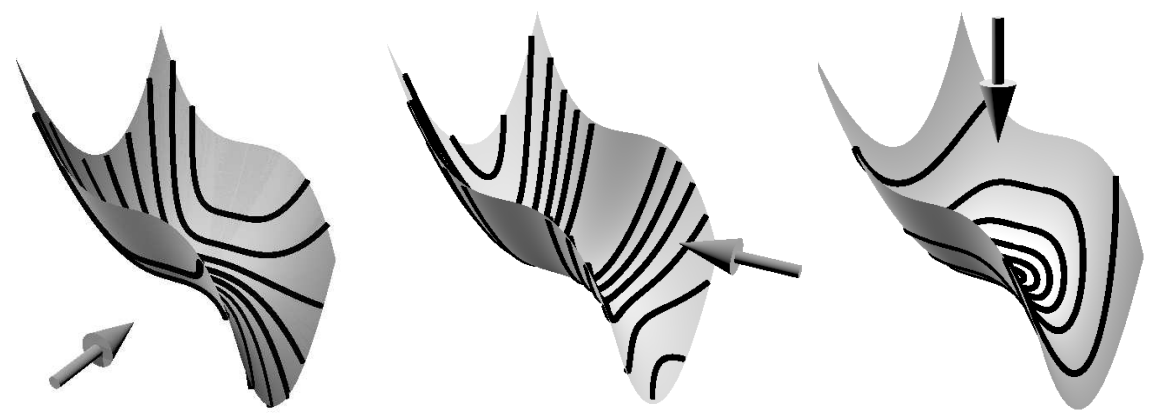

Fig. 1. Isophotes on a translational surface for different light directions d.

The second part of this observation is proved by solving the linear system

$$
\mathbf{p}_{u}(u, v)^{\top} \mathbf{n}(t)=0, \quad \mathbf{p}_{u}(u, v)^{\top} \mathbf{n}(t)=0
$$

for the surface parameters $(u, v)$, where $\mathbf{n}(t)$ is a quadratic rational parameterization of the spherical circle defined by (33), or a line in the plane which is perpendicular to $\mathbf{d}$.

In the case of developable quadratic polynomial surfaces (34), which are either planes, quadratic cylinders or quadratic half-cones, the two linear equations in (34) do not possess solutions for all $\mathbf{n}$. If solutions exist, then they are nonunique, and they form lines in the parameter domain. It turns out that the isophotes are either straight lines (for planes and cylinders) or half-lines (for half-cones).

Isophotes on surfaces with more general support functions can be parameterized by composing the parameterization of a spherical circle with the inverse Gauss map. If the support functions involves only rational operations and square roots, then one obtains square-root parameterizations of the isophotes.

Example 4. The isophotes on the translational surface from Example 1 have square-root parameterizations. Fig. 1 shows the isophotes for three different light directions.

\subsection{Other applications of the dual surface}

We conclude this section by briefly mentioning two additional applications of support functions and dual surfaces of free-form surfaces.

First, one may use them to compute the contours with respect to central projection. The tangent planes of the cone which is formed by the projection lines touching the surface correspond to a curve on the dual surface, which is simply the intersection with a plane. The intersection of this cone with the image plane 
gives the contour of the surface with respect to the central projection. In certain cases it is possible to find simple parameterizations of the planar intersections of the dual surfaces. E.g., in the case of quadratic polynomial surface, one obtains planar cubics on the dual surface which admit square-root parameterizations.

Second, it is an interesting idea to exploit duality for convex hull computation of free-form surfaces. This is described in more detail in [7].

\section{Support function approximation}

We propose a new method for computing isophotes on general free-form surfaces. The method consists of three steps. First, a piecewise quadratic approximation of the surface is generated. Second, this approximation is used to define a piecewise defined approximation of the inverse Gauss map of the surface. Finally, the Gauss map is applied to spherical circles.

\subsection{Piecewise quadratic approximation}

We consider a given surface $\mathbf{p}: \Omega \rightarrow \mathbb{R}^{3}$ with domain $\Omega=[0,1]^{2}$, which is assumed to be $C^{3}$ smooth, i.e., $\mathbf{p} \in C^{3}\left(\Omega, \mathbb{R}^{3}\right)$. Several techniques for generating a $C^{1}$ smooth piecewise approximation $\mathbf{p}^{*}$ exist.

For instance, one may use any triangulation of the domain and then use Powell-Sabin elements with respect to this triangulation, see [14]. The PowellSabin spline is uniquely determined by given first order Hermite data at the vertices of the original triangulation (which can be sampled from the given function), and it is $C^{1}$ smooth. However, Powell-Sabin elements tend to create relatively long and thin triangles, and therefore the visual quality of the approximation is often not satisfying.

Instead we use a piecewise quadratic function with respect to a criss-cross partition of the domain. First, the domain $\Omega$ is split into $n^{2}$ boxes with vertices $\left(\frac{i}{n}, \frac{j}{n}\right)_{i, j=0, \ldots, n}$. Second, each box is split into four triangles by adding the diagonals of the box. The space of piecewise quadratic functions, which are $C^{1}$ smooth, will be denoted with $S_{n}$. It is spanned by the translates the Zwart-Powell element, which is a special box spline, see [3].

Recently, a quasi-interpolation operator $Q$ for this space has been presented in [8]. For a given value of $n$, the operator $Q$ maps any function $f \in C^{3}(\Omega, \mathbb{R})$ into a piecewise quadratic approximation $Q f \in S_{n}$. When applied to the three components of the surface $\mathbf{p}$, one obtains a piecewise quadratic approximation $\mathbf{p}^{*}=Q \mathbf{p} \in S_{n}^{3}$. The computation of $Q \mathbf{p}$ requires solely the evaluations of $\mathbf{p}$ at certain points.

As shown in [8], this operator preserves quadratic functions and produces approximations $Q f$ which possess the optimal approximation order 3 with respect to the maximum norm in $C(\Omega, \mathbb{R})$. In addition, all first and second derivatives are approximated with order 2 and 1.

More precisely, the maximum difference between $\mathbf{p}$ and $\mathbf{p}^{*}$ and between its various first and second derivatives, can be bounded by $\hat{C}_{k} \Delta^{k}$, where $k=3,2,1$, respectively. The constants $\hat{C}_{k}$ depend on $\mathbf{p}$ and $\Delta=\frac{1}{n}$ is the size of the boxes. 


\subsection{Approximate dual implicitization}

Once a piecewise quadratic approximation $\mathbf{p}^{*}$ has been found, we use it to define an approximate inverse Gauss map.

1. First we split $\mathbf{p}^{*}$ into its polynomial segments. Each segment is represented as a quadratic triangular Bézier patch.

2. If one of these patches contains parabolic points, but is not entirely developable, then the corresponding points in the triangular domain form one or more (at most three) straight lines. These lines will be called the parabolic lines, see [2].

In this case we split the domain triangle along the parabolic lines and triangulate the resulting subdomains. After this step, all patches either do not contain any parabolic points in the interior of their domains, or they are developable surfaces. Let

$$
\left\{\mathbf{p}_{i}^{*}, i=1, \ldots, N\right\}
$$

be the set of the obtained patches.

3. We compute the Gauss images $G_{i} \subset \mathbb{S}^{2}$ of all patches $\mathbf{p}_{i}^{*}$.

- For non-developable patches we obtain curved spherical triangles, which are bounded by spherical conics, i.e., by intersections of quadratic cones (with their apices at the origin) with the unit sphere.

- If one of the patch boundaries is a parabolic line, then the Gauss image degenerates into a curved spherical biangle. Indeed, the normals of quadratic patches along their (at most 3) parabolic lines are constant. If $\mathbf{p}$ is regular, then also $\mathbf{p}^{*}$ is regular, provided that $\Delta$ is sufficiently small. Consequently, at most one the three patch boundaries is a parabolic line for each non-developable patch, since parabolic lines on quadratic patches intersect in singular points.

- The Gauss images of developable patches are arcs of spherical conics.

The collection of all Gauss images $G_{i}$ covers a certain subset of the unit sphere. Each point may be covered several times. Each Gauss images $G_{i}$ can be represented by the equations of (at most) three cones and by an auxiliary plane,

$$
G_{i}=\left\{\mathbf{n} \in \mathbb{S}^{2}: \mathbf{n}^{\top} A_{i}^{(1)} \mathbf{n} \geq 0 \wedge \mathbf{n}^{\top} A_{i}^{(2)} \mathbf{n} \geq 0 \wedge \mathbf{n}^{\top} A_{i}^{(3)} \mathbf{n} \geq 0 \wedge \mathbf{n}^{\top} \mathbf{w}_{i} \geq 0\right\}
$$

where the symmetric matrices $A_{i}^{(j)}$ represent the cones and the vector $\mathbf{w}_{i}$ is the normal vector of the auxiliary plane.

4. Finally we compute the inverse Gauss maps.

- For non-developable surface patches $\mathbf{p}_{i}^{*}$ we compute the rational support function $H_{i}$, see Section 2.2, and the inverse Gauss map $\Gamma_{i}=\nabla H_{i}$, see Eq. (27). $H_{i}$ and $\Gamma_{i}$ are rational functions of degree $3 / 2$ and $4 / 4$, respectively.

- The Gauss map $\Gamma_{i}$ of a developable surface patch $\mathbf{p}_{i}^{*}$ assigns to each normal in $G_{i}$ a line segment on the surface patch. 
The union of the support functions defines a multi-valued mapping

$$
H^{*}: \quad \bigcup_{i=1}^{N} G_{i} \rightarrow \mathbb{R}: \quad \mathbf{n} \mapsto \bigcup_{\mathbf{n} \in G_{i}}\left\{H_{i}(\mathbf{n})\right\}
$$

which approximates the support function of the original surface $\mathbf{p}$. The surface defined by $H^{*}(\mathbf{n})=1$, where we extended the domain of each support function segment to the cone $\mathbb{R} G_{i}$, can be seen as an approximate dual implicitization of the original surface, cf. (8). (See [6] for information on approximate implicitization.)

The union of the Gauss maps defines a multi-valued mapping

$$
\Gamma^{*}: \bigcup_{i=1}^{N} G_{i} \rightarrow \mathbb{R}^{3}: \quad \mathbf{n} \mapsto \bigcup_{\mathbf{n} \in G_{i}}\left\{\Gamma_{i}(\mathbf{n})\right\}
$$

which approximates the inverse Gauss map of the original surface $\mathbf{p}$.

\subsection{Isophote approximation}

We compute the isophotes on the approximating surface $\mathbf{p}^{*}$ for a given light direction $\mathbf{d}$ and different angles $\phi_{0}$. This is done by applying the mapping $\Gamma^{*}$ to the points of the corresponding circles $\mathbf{n}^{\top} \mathbf{d}=\cos \phi_{0}$ on the unit sphere. The circles are represented as rational quadratic curves and the intersections with the boundaries of the domains $G_{i}$ are found by numerically solving quartic equations.

Several examples are shown in Figures 2-4. It can be seen that the quality of the isophotes improves if a larger number of quadratic patches (and hence a smaller box size $\Delta$ ) is used.

We consider an arbitrary but fixed value of $\phi_{0} \in[0, \pi]$. The isophotes can be described with the help of the brightness functions

$$
\beta(u, v)=\mathbf{d}^{\top} \mathbf{N}(u, v) \quad \text { and } \quad \beta^{*}(u, v)=\mathbf{d}^{\top} \mathbf{N}^{*}(u, v)
$$

of $\mathbf{p}$ and $\mathbf{p}^{*}$, where $\mathbf{N}=\left(\mathbf{p}_{u} \times \mathbf{p}_{v}\right) /\left\|\mathbf{p}_{u} \times \mathbf{p}_{v}\right\|$ is the field of unit normals of the given surface $\mathbf{p}$ and $\mathbf{N}^{*}$, which is similarly defined, is the field of unit normals of the approximating surface $\mathbf{p}^{*}$. The level sets

$$
L=\beta^{-1}\left(\cos \phi_{0}\right) \quad \text { and } \quad L^{*}=\left(\beta^{*}\right)^{-1}\left(\cos \phi_{0}\right)
$$

in the parameter domain define the isophotes

$$
\mathbf{p}(L) \text { and } \mathbf{p}^{*}\left(L^{*}\right)
$$

on the exact and on the approximating surface, respectively.

For any two closed sets $A, B \subseteq \mathbb{R}^{k}$, let

$$
\operatorname{HD}(A, B)=\max _{\mathbf{x} \in A} \min _{\mathbf{y} \in B}\|\mathbf{x}-\mathbf{y}\|
$$

be their one-sided Hausdorff distance. We will use it to analyze the convergence of the isophotes. 

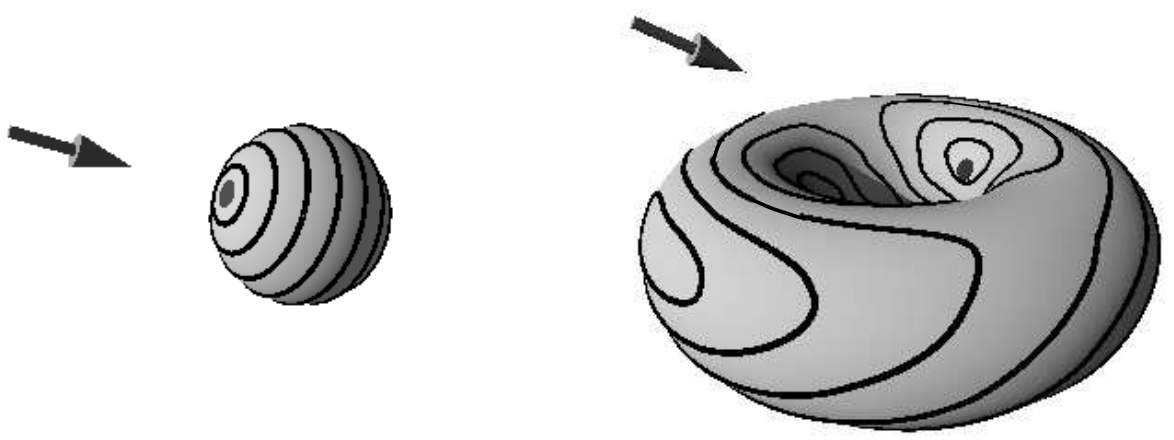

Fig. 2. The circles on the unit sphere are mapped into the isophotes of a quadratic approximation of a torus surface.
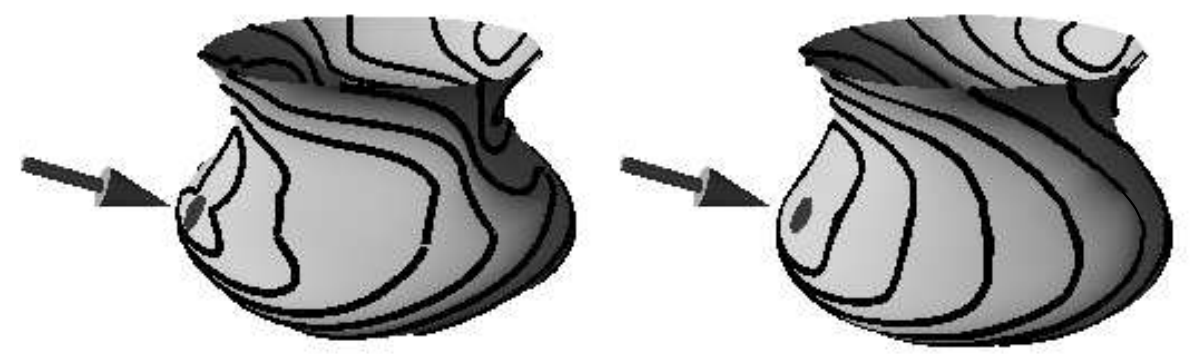

Fig. 3. Isophotes on a vase-shaped surface, which is approximated by 32 (left) and 100 (right) quadratic patches.

Theorem 1. Consider a $C^{3}$ smooth surface patch $\mathbf{p}$ whose domain $\Omega=[0,1]^{2}$ does neither contain parameter values of parabolic points of the surface nor points where the surface normal is parallel to the light direction $\mathbf{d}$. We approximate the surface using the quasi-interpolant described in Section 4.1 and use the result to compute the isophote for a given value of $\cos \phi_{0}$. There exists a constant $C$, which depends on the given surface $\mathbf{p}$, on the light direction $\mathbf{d}$ and the angle $\phi$, such that

$$
\max \left\{\operatorname{HD}\left(\mathbf{p}(L), \mathbf{p}^{*}\left(L^{*} \cup \partial \Omega\right)\right), \operatorname{HD}\left(\mathbf{p}^{*}\left(L^{*}\right), \mathbf{p}(L \cup \partial \Omega)\right)\right\} \leq C \Delta^{2}
$$

where $\Delta$ is the box size used for the quasi-interpolants.

Proof. The brightness functions $\beta$ and $\beta^{*}$ are $C^{2}$ smooth and merely continuous, respectively. However, $\beta^{*}$ is piecewise differentiable (within each triangle of the criss-cross triangulation). Since the first derivatives of $\mathbf{p}^{*}$ have uniform quadratic convergence, we may conclude that there exists a constant $C_{1}$ such that

$$
\forall(u, v) \in \Omega: \quad\left|\left(\beta-\beta^{*}\right)(u, v)\right|<C_{1} \Delta^{2} .
$$




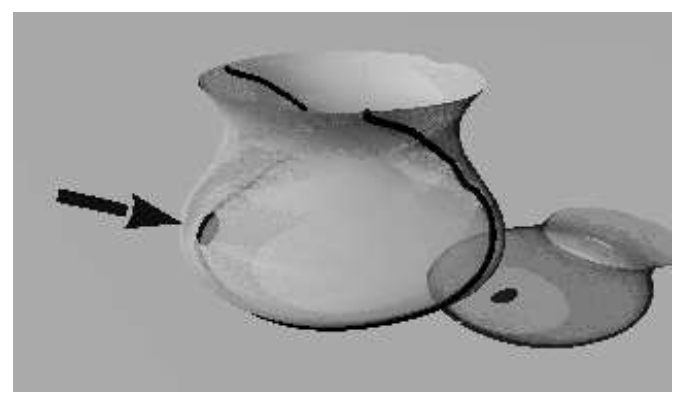

Fig. 4. The contour generator of a vase approximated by 100 quadratic patches and its image under the parallel projection. The contour generator is projected into the contour of the surface.

Since parabolic points and points with normal $\mathbf{d}$ were excluded from $\Omega$, the length of the gradient $\nabla \beta$ (with respect to the surface parameters $u, v$ ) is strictly positive, i.e., there exists a constant $C_{2}$ such that

$$
\forall(u, v) \in \Omega: \quad\|(\nabla \beta)(u, v)\|>C_{2} .
$$

As the first and second derivatives of the quadratic approximation converge to the derivatives of $\mathbf{p}$, we obtain that

$$
\forall(u, v) \in \Omega^{0}: \quad\left\|\left(\nabla \beta^{*}\right)(u, v)\right\|>\frac{C_{2}}{2}
$$

for sufficiently small values of $\Delta$, where $\Omega^{0}$ is the domain which is obtained by excluding the lines of the criss-cross triangulation.

In order to prove (42) for the first of the two one-sided Hausdorff distances, we consider an arbitrary point $\left(u_{0}, v_{0}\right)$ in the parameter domain which belongs to the level set $L$. The value of the brightness function $\beta^{*}$ at this point satisfies

$$
\cos \phi_{0}-C_{1} \Delta^{2} \leq \beta^{*}\left(u_{0}, v_{0}\right) \leq \cos \phi_{0}+C_{1} \Delta^{2} .
$$

Starting at this point, we create a curve $\mathbf{c}(s)$ by integrating the normalized gradient field $\nabla \beta^{*} /\left\|\nabla \beta^{*}\right\|$. This curve is then given by an arc length parameterization, since it is the integral curve of a field of unit vectors. While it is not $C^{1}$ smooth (as the gradient $\nabla \beta^{*}$ are not $C^{1}$ ), it is $C^{1}$ except for its intersections with the lines of the criss-cross triangulation. The restriction $\left(\beta^{*} \circ \mathbf{c}\right)(s)$ of the brightness function $\beta^{*}$ to this curve defines a continuous and monotonic function of the arc length parameter. Its derivative with respect to $s$ - which is defined everywhere except for the intersections with the lines in the criss-cross triangulation - satisfies

$$
\left(\beta^{*} \circ \mathbf{c}\right)^{\prime}(s)=\frac{\left(\nabla \beta^{*}\right)(\mathbf{c}(s))}{\left\|\left(\nabla \beta^{*}\right)(\mathbf{c}(s))\right\|} \cdot\left(\nabla \beta^{*}\right)(\mathbf{c}(s))=\left(\nabla \beta^{*}\right)(\mathbf{c}(s))>\frac{C_{2}}{2} .
$$

If $\beta^{*}\left(u_{0}, v_{0}\right)<\cos \phi_{0}$, then we travel along the curve $\mathbf{c}(s)$ into the direction of the gradient, which increases the value of $\beta^{*}$, until we hit either the level set $L^{*}$ 
or the domain boundary $\partial \Omega$. However, since the derivative satisfies (47) and the deviation of $\beta^{*}\left(u_{0}, v_{0}\right)$ from $\cos \phi_{0}$ is not larger than $C^{1} \Delta^{2}$, the travel distance is bounded by

$$
C_{1} \Delta^{2} \frac{2}{C_{2}}
$$

Otherwise, if $\beta^{*}\left(u_{0}, v_{0}\right)>\cos \phi_{0}$, then we travel along the curve $\mathbf{c}(s)$ into the direction of the negative gradient and use a similar argument to bound the travel distance.

Summing, we find a point $(u, v) \in L^{*} \cup \partial B$ such that

$$
\left\|(u, v)-\left(u_{0}, v_{0}\right)\right\| \leq C_{1} \Delta^{2} \frac{2}{C_{2}} .
$$

Now we apply the mappings $\mathbf{p}$ and $\mathbf{p}^{*}$ and use the triangle inequality,

$$
\left\|\mathbf{p}^{*}(u, v)-\mathbf{p}\left(u_{0}, v_{0}\right)\right\| \leq\left\|\mathbf{p}^{*}(u, v)-\mathbf{p}(u, v)\right\|+\left\|\mathbf{p}(u, v)-\mathbf{p}^{*}\left(u_{0}, v_{0}\right)\right\| .
$$

The first term on the right hand side is bounded by $C_{3} \Delta^{3}$, due to the third order of approximation. The second term is bounded by $2 C_{4}\left(C_{1} / C_{2}\right) \Delta^{2}$, where $C_{4}$ is a global bound on the norm of the Jacobian of $\mathbf{p}$. This proves the result for the first Hausdorff distance in (42). The second Hausdorff distance can be dealt with similarly, but this time using the smooth integral curves of the vector field $\nabla \beta /\|\nabla \beta\|$.

\section{Conclusion}

We discussed the support functions of several special classes of free-form surfaces. Based on observations concerning quadratic polynomial surfaces, which possess rational support functions of degree $3 / 2$, and on a quasi-interpolation scheme for bivariate quadratic splines, we formulated an algorithm for approximate isophote computation and analyzed its convergence.

Instead of quadratic polynomial surfaces, one might use other classes of approximating surfaces which still admit closed-form representations of the inverse Gauss maps (e.g., using square roots). This could give approximations schemes for isophotes with an even higher rate of convergence, which may be a possible topic of further research.

Another possible application of our results is mesh contour smoothing, see [27]. This motivates the further investigation of surfaces with simple contours, such as quadratic polynomial surfaces.

Acknowledgement. M. Aigner and B. Jüttler gratefully acknowledge financial support by the Austrian Science Fund through the National Research Network S92 "Industrial Geometry" and by grant WTZ 11/2007 of the Austrian-Spanish programme for scientific cooperation. The second author acknowledges the support of the Spanish grant MTM2008-04699-C03-03. M. L. Sampoli acknowledges the financial support of the Italian GNCS through the "Senior visiting program 2008". 


\section{References}

1. Aigner, M., Jüttler, B., Gonzalez-Vega, L., Schicho, J.: Parameterizing surfaces with certain special support functions, including offsets of quadrics and rationally supported surfaces, J. Symb. Comp. 44, 180-191 (2009)

2. Bastl, B. Jüttler, B., Kosinka, J., Lávička, M., Computing exact rational offsets of quadratic triangular Bézier surface patches, Comp.-Aided Design 40, 197-209 (2008).

3. de Boor, C, Höllig. K., Riemenschneider, S.: Box Splines, Springer (1993).

4. Degen, W.L.F.: The types of triangular Bézier surfaces. In Mullineux, G. (editor): The Mathematics of Surfaces IV, volume 38 of The Institute of Mathematics and its Applications Conference Series, Clarendon Press, Oxford, 153-171 (1996)

5. Dietz, R., Hoschek, J., Jüttler, B., An algebraic approach to curves and surfaces on the sphere and on other quadrics. Comp. Aided Geom. Design 10, 211-229 (1993)

6. Dokken, T.: The GAIA project on intersection and implicitization, in: Jüttler, B., Piene, R., Geometric Modeling and Algebraic Geometry, Springer, 5-30 (2008).

7. Elber, G., Johnstone, J.K., Kim, M.-S., Seong, J.-K.: The Kernel of a Freeform Surface and its duality with the Convex Hull of its Tangential Surface. Int. J. Shape Modeling 12, 129-142 (2006)

8. Foucher, F., Sablonnière, P.: Approximating partial derivatives of first and second order by quadratic spline quasi-interpolants on uniform meshes. Mathematics and Computers in Simulation 77, 202-208 (2008).

9. Gravesen, J. : Surfaces parametrised by the normals, Computing 79, 175-183 (2007)

10. Gravesen, J., Jüttler, B., Šrr, Z.: On Rationally Supported Surfaces, Comp. Aided Geom. Design 25, 320-331 (2008).

11. Hahmann, S.: Visualization techniques for surface analysis. in: Bajaj, C. (ed.): Advanced Visualization Techniques, Wiley, 49-74 (1999)

12. Hahmann, S., Belyaev, A., Buse, L., Elber, G., Mourrain, B., Rössl, C.: Shape Interrogation. In: Floriani, L., Spagnuolo, M. (eds.): Shape Analysis and Structuring, Springer, 1-52 (2007).

13. Hoschek, J: Dual Bézier curves and surfaces, in: Barnhill, R.E., Boehm, W.: Surfaces in Computer Aided Geometric Design, North Holland, 147-156 (1983)

14. Hoschek, J., Lasser, D.: Fundamentals of Computer Aided Geometric Design. AK Peters, Wellesley (1993).

15. Johansen, H., Løberg, M., Piene, R.: Monoid hypersurfaces. In: Jüttler, B., Piene, R.: Geometric Modeling and Algebraic Geometry, Springer, 55-78 (2008)

16. Lávička, M., Bastl, B., Rational hypersurfaces with rational convolutions, Comp. Aided Geom. Design 24, 410-427 (2007).

17. Lichtenauer, J., Hendriks, E., Reinders, M.: Isophote properties as features for object detection. Proc. IEEE Conf. Computer Vision and Pattern Recognition, vol. 2, 649-654 (2005).

18. Morse, B. S., Schwartzwald, D.: Isophote-based interpolation. Proc. Int. Conference on Image Processing, vol. 3, 227-231 (1998)

19. Mühlthaler, H., Pottmann, H.: Computing the Minkowski sum of ruled surfaces, Graphical Models 65, 369-384 (2003)

20. Peternell, M., Pottmann, H.: A Laguerre geometric approach to rational offsets. Comput. Aided Geom. Design 15, 223-249 (1998)

21. Peters, J., Reif, U., The 42 equivalence classes of quadratic surfaces in affine $n$ space, Comp. Aided Geom. Design 15, 459-473 (1998) 
22. Poeschl, T.: Detecting surface irregularities using isophotes. Comput. Aided Geom. Design 1, 163-168 (1984).

23. Pottmann, H.: Rational curves and surfaces with rational offsets, Computer Aided Geometric Design 12, 175-192 (1995)

24. Pottmann, H., Lü, W., Ravani, B.: Rational Ruled Surfaces and Their Offsets, Graphical Models and Image Processing 58, 544-552 (1996)

25. Pottmann, H., Steiner, T., Hofer, M., Haider, C., Hanbury, A.: The Isophotic Metric and its Application to Feature Sensitive Morphology on Surfaces, in: Pajdla, T., Matas, J. (eds.): Proc. ECCV, Part IV, Springer, Lecture Notes in Computer Science vol. 3024, 560-572 (2004).

26. Sabin, M.: A Class of Surfaces Closed under Five Important Geometric Operations, Report no. VTO/MS/207, British aircraft corporation, available at http://www.damtp.cam.ac.uk/user/na/people/Malcolm/vtoms/vtos.html (1974)

27. Wang, L., Tu, C. H., Wang, W., Meng, X.X., Chan, B., Yan, D.M.: Silhouette smoothing for real-time rendering of mesh surfaces, IEEE Trans. on Vis. Comp. Graphics 14, 640-652 (2008).

28. Zhan, Y., Wang M., Li M.: An Isophote-Oriented Image Interpolation Method. Proc. Int. Symp. Computer Science and Computational Technology, 723-726 (2008). 